\title{
Appreciation to Reviewers
}

With this issue, the Journal of Behavioral Health Services \& Research (JBHS\&R) concludes its 48th year of publication. I am very grateful to all of the authors, reviewers, and $J B H S \& R$ Editorial Board members for their exceptional contributions to this peer-reviewed publication, particularly during this COVID-19 Global Pandemic.

Editing and publishing a scholarly peer-reviewed journal is an amazing as well as complex initiative that involves working with a critical mass of fascinating, highly creative, well-educated, and very productive assemblage of superb individuals. Certainly, the $J B H S \& R$ could not exist without the manuscript authors, the $J B H S \& R$ Editorial Board members, and the Ad Hoc External Manuscript Reviewers.

At this time, I would like to recognize and express my profound appreciation to the many individuals who continue to support this scholarly publication: Mr. Chuck Ingoglia and Ms. Mary Johnson at the National Council for Mental Wellbeing; Ms. Katherine Chabalko, Mr. Bob Darnowsky, Mr. Arene Ligo, Mr. Genesis Obero, Mr. Soren Malpass, and Ms. Sara Yanny-Tillar at Springer publishing; Dr. Mario Hernandez, Dr. Ardis Hanson, Ms. Sarah Sheffield, and Ms. Kimberly Menendez at the University of South Florida; and the academic and institutional libraries who have electronic access to the $J B H S \& R$.

This scholarly publication is committed to publishing applied services research, services delivery, policy, and implementation science manuscripts in behavioral health (including alcohol, drug use, and mental health) services. The ability to improve the quality of the manuscripts published in the $J B H S \& R$ is largely dependent upon the strength of the double-blind peer-review process. Manuscript referees provide the $J B H S \& R$ with an immeasurable wealth of expertise and willingness to volunteer their time and vivacity on behalf of the $J B H S \& R$. Therefore, I especially would like to thank and express my deepest appreciation to the individuals listed below, all of whom kindly reviewed at least one manuscript submitted to the $J B H S \& R$ during 2020-2021.

Bruce Lubotsky Levin, DrPH, MPH

Editor-in-Chief

Journal of Behavioral Health Services \& Research

University of South Florida

Melissa Abraham
Martha Aby
Christina Aivadyan
Becci Akin
Alexandra Albizu-Jacob
Paige Alitz

Melissa Abraham

Christina Aivadyan

Becci Akin

Paige Alitz

Erica Anderson
Cynthia L. Arfken
Dan Aune
Melissa Azur
William Bane
Jim Banta

Emily Baton

Rachel Battaglia

Christy L. Beaudin

Astrid Beigel

Vivian Bell

Sean Bennett 


\begin{tabular}{|c|c|c|}
\hline Rosalyn M. Bertram & T. Freeman Gerhardt & Norma Mtume \\
\hline Casey Bohrman & Affan Ghaffari & Hans Oh \\
\hline Jason E. Boye & Brent J. Gibbons & Howard Padwa \\
\hline Benjamin Brady & Paul B. Gold & Vierne Placide \\
\hline Eileen Brennan & Amy L Green & Esra Polat \\
\hline Laura Brennan & Catherine Greeno & Michele Preyde \\
\hline Eric J. Bruns & Christine E. Grella & Scott E. Provost \\
\hline Donna L. Burton & Keri J. Griffin & Jason Raines \\
\hline Linda M Callejas & Ardis Hanson & Sharon Reif \\
\hline Jeff Capobianco & Eric R. Hardiman & Vestena Robbins \\
\hline Humberto López Castillo & Indira Harris & Craig S. Rosen \\
\hline Melody Chavez & Linda Henderson-Smith & Monica Rousseau \\
\hline Johnson Chun-Sing Cheung & Michael Hendryx & Deborah Scharf \\
\hline Beomyoung Cho & Cole Hooley & Claudia Sellmaier \\
\hline Joy Chudzynski & Laura Hume & Amanda Sharp \\
\hline Michael Clarkson-Hendrix & Heidi Israel & E Mackenzie Shell \\
\hline Mariah Coe & Siavash Jafari & Rachel Shelton \\
\hline Sara Coffey & Vandana Joshi & Prabin Shrestha, \\
\hline Donna Cohen & Jamie Kammer & Monica Simmonds \\
\hline Edlin Garcia Colato & Jody Kamon & David Sommerfeld \\
\hline Erin Comartin & Lourah Kelly & Christina Studts \\
\hline Joseph Comaty & Suzanne Kerns & Faye S Taxman \\
\hline Alexander Cowell & Vanessa V Klodnick & Judith L. Teich \\
\hline Lee A. Crandall & Derrick Kranke & Melba Hernandez Tejada \\
\hline M. Lynn Crismon & Marsha Langer Ellison & James M. Topolski \\
\hline Gracelyn Cruden & Thomas LaPorte & Jennifer Tran \\
\hline James J. Dahl & Mary Jo Larson & Lacey Tucker \\
\hline Bruce R. DeForge & Xiaoxue Li & Enya B. Vroom \\
\hline Maridelys Detres & Robert Lucio & Neal Wallace \\
\hline Ann Dirks-Linhorst & Ami Lynch & Christine M. Walrath-Greene \\
\hline Karyn Dresser & Michael Anthony Mancini & Wendy Ward \\
\hline Michelle Duda & Stephanie Marhefka & Suzanne Wenzel \\
\hline John Encandela & Joe Marrone & Svetlana Yampolskaya \\
\hline Richard A. Epstein & Jason Matejkowski & Michael Yogman \\
\hline Noe Erazo & Dennis McCarty & Anna Zamora-Kapoor \\
\hline Ekom Essien & Colleen E McKay & Huaiyu Zang \\
\hline James H Ford & Charles Mendez & Amanda Zavodnick \\
\hline Carrie Fry & Kimberly Menendez & Hui Zhang \\
\hline Dainelys Garcia & Christopher G. Mitchell & \\
\hline
\end{tabular}

Publisher's Note Springer Nature remains neutral with regard to jurisdictional claims in published maps and institutional affiliations. 